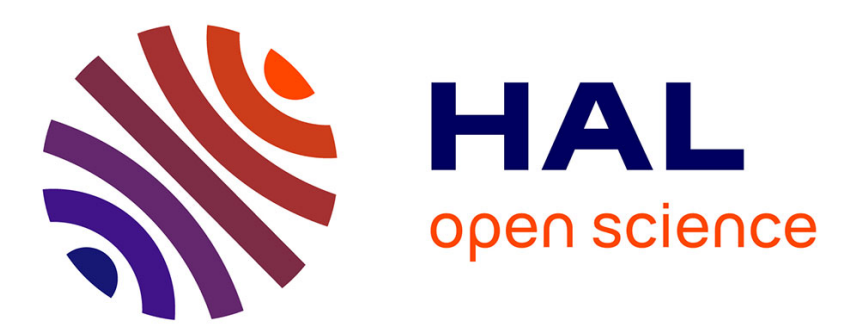

\title{
Simulation des trajectoires d'électrons secondaires pour le test des circuits intégrés au microscope électronique à balayage
}

\author{
F. Roche, R. Barillé
}

\section{- To cite this version:}

F. Roche, R. Barillé. Simulation des trajectoires d'électrons secondaires pour le test des circuits intégrés au microscope électronique à balayage. Journal de Physique III, 1992, 2 (11), pp.2155-2163. 10.1051/jp3:1992237 . jpa-00248873

\section{HAL Id: jpa-00248873 https://hal.science/jpa-00248873}

Submitted on 1 Jan 1992

HAL is a multi-disciplinary open access archive for the deposit and dissemination of scientific research documents, whether they are published or not. The documents may come from teaching and research institutions in France or abroad, or from public or private research centers.
L'archive ouverte pluridisciplinaire HAL, est destinée au dépôt et à la diffusion de documents scientifiques de niveau recherche, publiés ou non, émanant des établissements d'enseignement et de recherche français ou étrangers, des laboratoires publics ou privés. 
Classification

Physics Abstracts

$41.90-02.60$

\title{
Simulation des trajectoires d'électrons secondaires pour le test des circuits intégrés au microscope électronique à balayage
}

\author{
F. M. Roche et R. Barillé \\ Laboratoire d'Informatique, de Robotique et de Microélectronique de Montpellier (LIRMM), \\ URA CNRS DO1480, Université de Montpellier II : Sciences et Techniques du Languedoc, \\ Pl. E. Bataillon, 34095 Montpellier Cedex, France
}

(Reçu le 16 mars 1992, accepté le 21 juillet 1992)

\begin{abstract}
Résumé. - La nécessité d'accéder au potentiel effectif de pistes enterrées de circuits intégrés lors du test par faisceau d'électrons nous a conduit à analyser les différents facteurs déterminant la mesure. Un de ceux-ci est le trajet suivi par l'électron secondaire de la surface irradiée au détecteur, en présence d'une polarisation des pistes. Ce papier présente une simulation des trajectoires des électrons secondaires en environnement de test (dose incidente faible, circuit passivé, analyseur dans la colonne). L'évolution des trajectoires en fonction du champ d'extraction et des niveaux de polarisation est analysée et reliée au courant effectivement détecté. Le logiciel permet aussi d'étudier la modification de temps de transit provoquée par un changement rapide du potentiel de la piste.
\end{abstract}

\begin{abstract}
The requirement to access the effective potential of buried lines during electron beam testing operation yields us to analyse the different impediments involved in the measurement. One of these is the secondary electron pathlength from the irradiated surface to the detector, under different track biasing configurations. This paper summarizes a simulation of the secondary electron trajectories in a testing environment (low incident dose, passivated device, in the lens analyzer). The development of trajectories according to the extraction voltage and the polarization levels is analyzed and related to the effectively measured current in the detector. The software allows us to acknowledge the «transit time » alteration induced by a fast change in the track potential.
\end{abstract}

\section{Introduction.}

La réduction de dimension des circuits intégrés jointe à une complexité croissante des dispositifs appellent de nouveaux concepts pour tester le fonctionnement interne des V.L.S.I. ou faire le diagnostic de leurs défaillances. Le test par faisceau d'électrons (EBT) est de ceuxlà $[1,2]$. Il met en œuvre la spectroscopie d'électrons secondaires pour réaliser en temps réel l'image des potentiels électriques locaux du dispositif. A cet effet, le pinceau d'électrons est utilisé à la manière d'une sonde de très faible diamètre et de grande impédance, extrêmement mobile dont la mise en cuvre ne charge pas le circuit sous test par des effets capacitifs parasites [3].

En raison de ces spécificités le testeur à faisceau d'électrons se présente comme un outil particulièrement performant et bienvenu pour l'examen par contraste de potentiel de circuits 
microniques et submicroniques, notamment ceux de nouvelle génération à niveaux multiples d'interconnexions.

\section{Contexte de l'étude.}

Deux méthodes peuvent être utilisées pour le test de circuits passivés. La première consiste à ôter la couche d'oxyde de passivation pour accéder aux pistes enterrées sous l'oxyde. Outre son coût, ce contrôle à caractère destructif, ne peut trouver sa place dans un processus de fabrication industriel, si ce n'est pour une vérification statistique.

La seconde solution consiste à utiliser la méthode du contraste de potentiel par couplage capacitif (CCVC) qui permet une observation immédiate et, dans certaines conditions, non invasive des dispositifs en fonctionnement [4]. Elle met à profit le principe physique d'un couplage capacitif qui peut apparaitre en cours d'irradiation entre la surface de l'oxyde supérieur exposé au faisceau d'électrons et une piste enterrée soumise à une variation de potentiel [5]. Indirectement, le CCVC permet d'accéder à l'état électrique de cette piste.

Le constraste de potentiel se révèle donc particulièrement bien adapté à l'observation des circuits de plus en plus intégrés. Il est actuellement le seul qui permette l'accès au potentiel des couches enterrées de circuits multi-niveaux. Cependant en raison même de son caractère indirect, le CCVC présente encore un certain nombre de facteurs limitatifs. L'interprétation des résultats nécessite une maîtrise des différents paramètres expérimentaux impliqués dans la mesure. Outre l'interaction faisceau/circuit traitée par ailleurs [6-8] les facteurs essentiels sont les paramètres d'irradiation, l'introduction de singularités de potentiel et les conditions de collection et d'analyse des électrons secondaires réémis.

Une fois optimisées les incertitudes expérimentales d'accès à la mesure, les erreurs systématiques liées à la méthode seront abordées. La simulation réalisée ci-après a pour but de minimiser les premières.

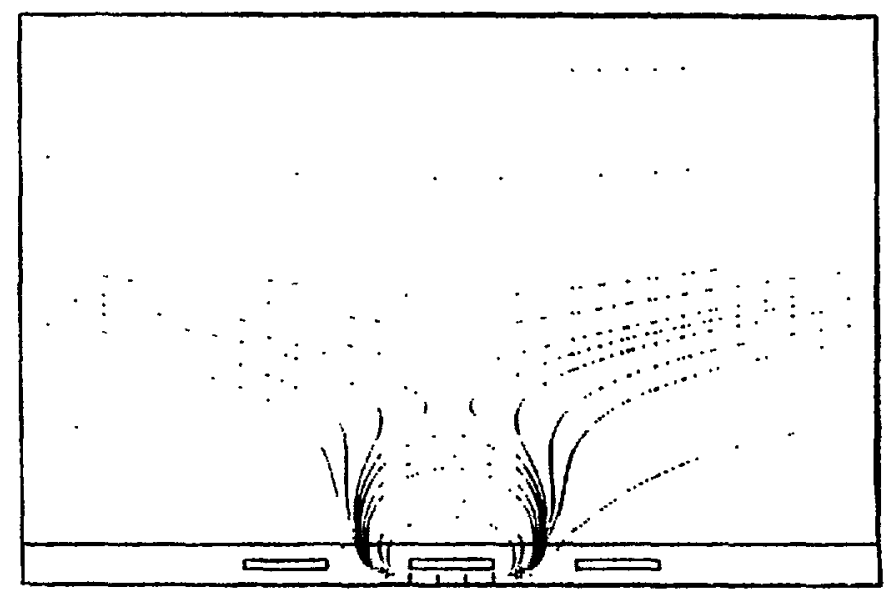

Fig. 1. - Tracé de lignes équipotentielles pour une largeur de piste $W=3 \mu \mathrm{m}$ et une distance de séparation entre les pistes $D=3 \mu \mathrm{m}$. La profondeur des pistes est de $1 \mu \mathrm{m}$. Le champ d'extraction est de $80 \mathrm{~V} / \mathrm{mm}$, Le potentiel minimum $V_{\min }=1,7 \mathrm{~V}$.

[Equipotential line distribution for a line width $W=3 \mu \mathrm{m}$ and a spacing between the lines $D=3 \mu \mathrm{m}$. The lines deep is $1 \mu \mathrm{m}$. The extraction field is $80 \mathrm{~V} / \mathrm{mm}$. The minimum potential $\left.V_{\min }=1.7 \mathrm{~V}.\right]$ 
1. Les conditions d'irradiation - L'énergie du faisceau primaire est faible (typiquement 1 à $1,5 \mathrm{keV}$ ) choisie dans la gamme d'existence du contraste de potentiel, c'est-à-dire en dessous de second cross-over EpII dans un domaine où le rendement est supérieur à $1[4,5]$. Le courant de faisceau est haché à l'aide d'un blanker de façon à limiter le phénomène d'accumulation de charge en surface.

2. La présence de singularités de potentiel — La présence dans le circuit de discontinuités de polarisation induit à la surface de l'échantillon des singularités dans le réseau équipotentiel. Ces dernières sont conditionnées à la fois par la configuration géométrique des pistes et par leurs états électriques. Dans certaines configurations particulières une inversion du champ peut même se produire et constituer pour les électrons réémis une barrière de potentiel (Fig. 1). Cet effet de champs locaux conditionne la collection des secondaires réémis et donc le courant total collecté. Il fait l'objet d'une modélisation.

3. La collection des électrons secondaires - Un moyen d'augmenter le rapport signal sur bruit est d'accroître le rendement de collection des électrons secondaires en plaçant au voisinage du dispositif (à quelques $\mathrm{mm}$ ) une grille d'extraction portée à un potentiel positif (de quelques volts à quelques milliers de volts). Si la présence de cette grille améliore considérablement la collection des électrons secondaires, elle n'en modifie pas moins la répartition des champs en général et des champs locaux en particulier, donc la hauteur de barrière et le courant collecté. C'est cet aspect qui a fait l'objet de l'étude et des simulations qui suivent.

4. L'analyse de l'énergie des électrons secondaires - Cette analyse spectroscopique est effectuée à l'aide d'une grille retardatrice portée à un potentiel convenable et placé dans la colonne au-dessus de la grille d'extraction [9]. Dans ce dispositif « in the lens » la distance inter-grille est augmentée et donc la résolution de la mesure s'en trouve accrue. Le dispositif (grille retardatrice) est asservi durant la mesure pour laisser passer un nombre d'électrons constant. On a alors $\Delta V_{\text {circuit }}=\Delta V_{\text {grille retardatrice fournie par la boucle de contre-réaction. Il }}$ permet d'effectuer une calibration en positionnant le faisceau sur des potentiels de référence.

\section{Modélisation du champ et simulation des trajectoires d'électrons.}

Afin d'évaluer l'incertitude sur le potentiel de surface à mesurer pour chaque configuration choisie ainsi que l'influence de la tension d'extraction sur la répartition des équipotentielles audessus du circuit, le potentiel a besoin d'être connu en tout point du domaine. Le logiciel calcule la modification de la distribution du potentiel créée par la grille d'extraction et par le potentiel des pistes. Le calcul est fait au-dessus de la structure et à l'intérieur de celle-ci. En particulier, il permet de déterminer le potentiel à la surface (Fig. 2). Pour cela l'espace est discrétisé et l'équation de Poisson est résolue en chacun des points du domaine par la méthode des différences finies [10].

$$
\varepsilon_{x}(x, y) \frac{\partial^{2} V}{\partial x^{2}}+\varepsilon_{y}(x, y) \frac{\partial^{2} V}{\partial y^{2}}=\rho(x, y) .
$$

Les conditions limites sur le bord du domaine sont prises en compte quels que soient les configurations choisies et le champ d'extraction utilisé. Elles sont imposées pour les frontières des différents conducteurs (condition de Dirichlet) et de la grille d'extraction. Le champ sortant est pris nul sur les limites verticales du domaine. Le système donné par l'équation de Laplace est tridiagonal. On le résout par la méthode du double balayage de Choleski avec une précision convenable. La distribution du potentiel au-dessus du dispositif (Fig. 1) permet alors la simulation des trajectoires des électrons secondaires émis du matériau afin d'étudier l'influence de divers paramètres sur leur collection. 


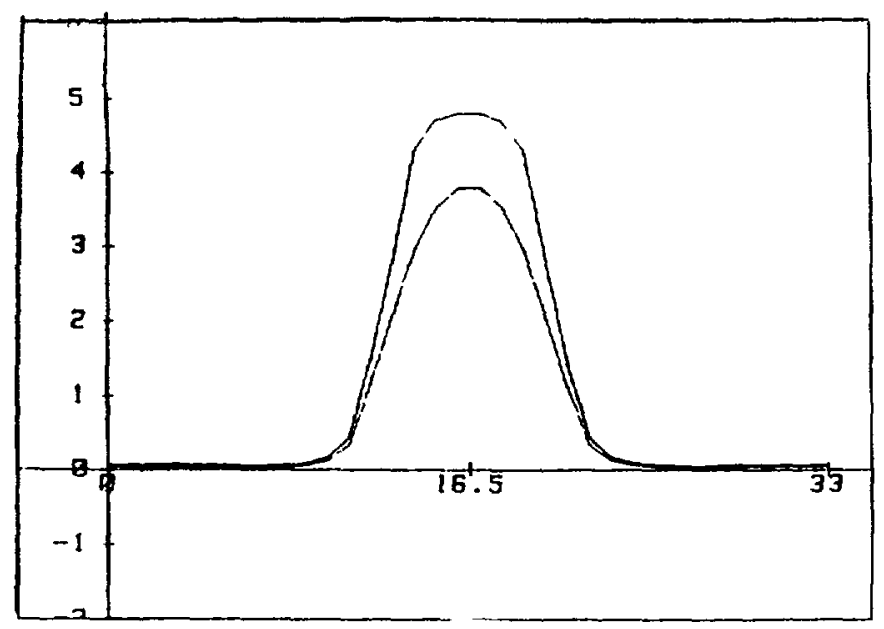

Fig. 2. - Distribution du potentiel de surface $V_{s}$ pour une configuration avec $W=5 \mu \mathrm{m}, D=3 \mu \mathrm{m}$, pour deux profondeurs de $0,8 \mu \mathrm{m}$ et $2,4 \mu \mathrm{m}$. Le champ d'extraction est de $20 \mathrm{~V} / \mathrm{mm}$.

[Surface potential distribution $V_{\mathrm{s}}$ for a configuration with $W=5 \mu \mathrm{m}, D=3 \mu \mathrm{m}$, and two depths of line $0.8 \mu \mathrm{m}$ and $2.4 \mu \mathrm{m}$ respectively. The Extraction field is $20 \mathrm{~V} / \mathrm{mm}$.]

La force électrique est connue aux sommets du maillage grâce au potentiel. Une double interpolation permet de déterminer en un point quelconque intérieur les deux composantes de l'accélération [11].

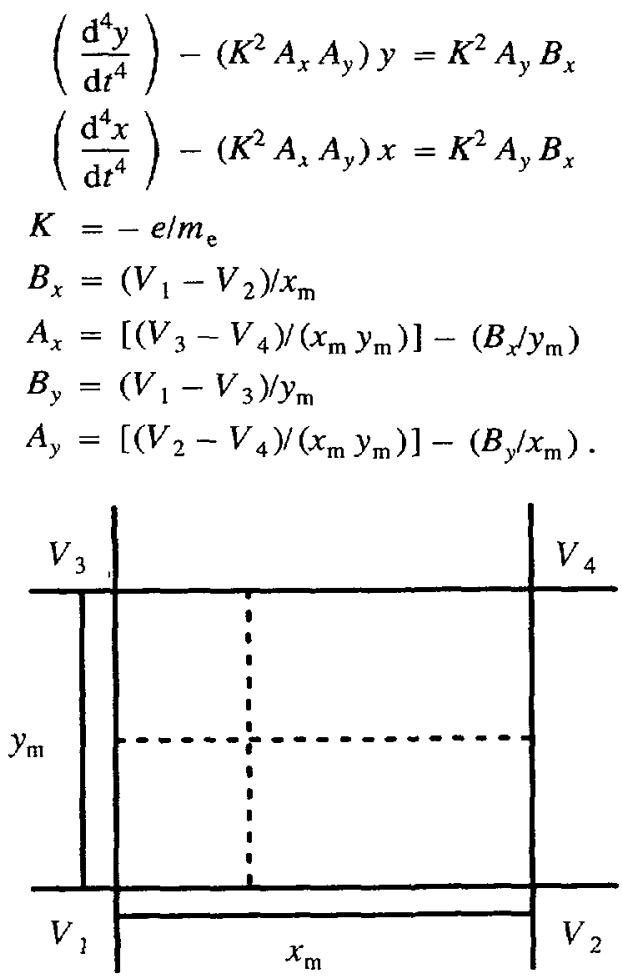


La position et la vitesse de l'électron dans chaque maille sert alors de condition initiale pour le pas suivant. De plus lorsque le courant circulant dans les pistes est important il se crée un champ magnétique qui modifie la trajectoire des électrons et peut être apprécié par les résultats des simulations de trajectoires des électrons (Fig. 3).
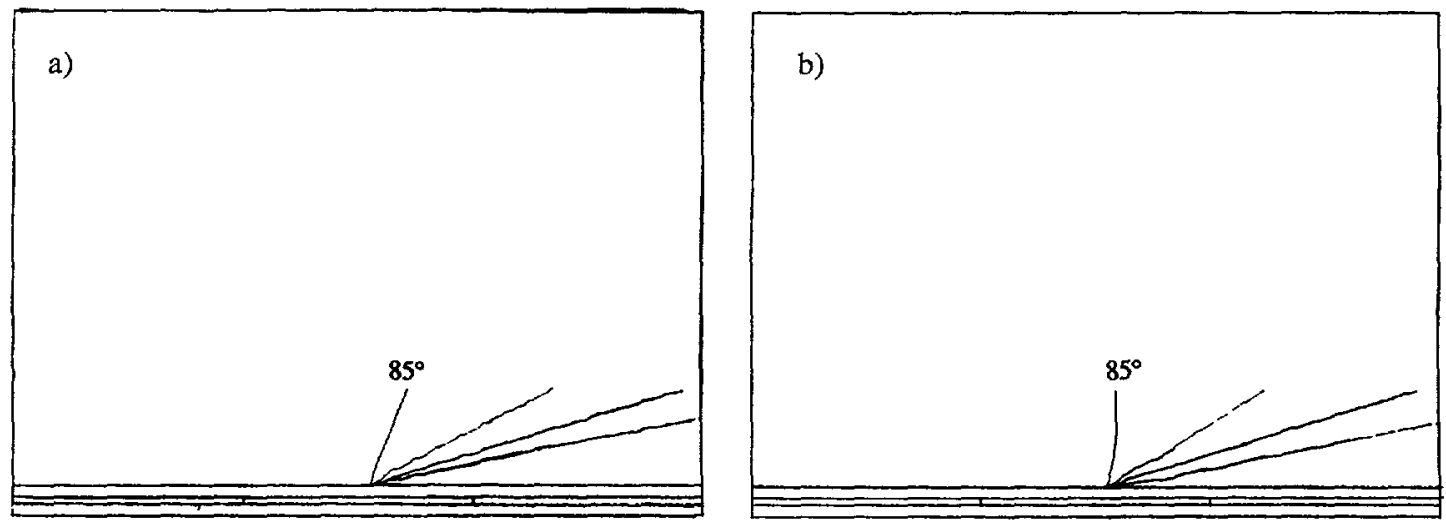

Fig. 3. - a) Trajectoires des électrons secondaires pour un champ magnétique correspondant à un courant de $1 \mathrm{~A}$, b) sans champ magnétique. Les variables sont l'angle de sortie des électrons $25^{\circ}, 45^{\circ}$, $65^{\circ}, 85^{\circ}$ et leur énergie $4 \mathrm{eV}$.

[a) Secondary electron trajectories for a magnetic field corresponding to a 1 A current, b) without magnetic field. The variables are the electron emission angles $25^{\circ}, 45^{\circ}, 65^{\circ}, 85^{\circ}$ and their energy $4 \mathrm{eV}$.]

\section{Conséquence des simulations.}

L'observation et la mesure du potentiel des différents niveaux de métallisation des circuits montre qu'il est indispensable d'utiliser un faible champ d'extraction sur les circuits passivés, alors qu'un fort champ est nécessaire pour les circuits dépassivés. De plus durant l'expérience une accumulation de charges en surface perturbe les mesures en modifiant la valeur du potentiel de surface. Il est donc important de comprendre ce phénomène et d'expliquer son mécanisme.

En présence du champ d'extraction, la différence de polarisation électrique des pistes enterrées provoque localement une barrière de potentiel que les électrons doivent franchir avant d'être accélérés. La figure 1 illustre ce passage des lignes équipotentielles par un minimum. Cette barrière de potentiel $U_{\mathrm{so}}=V_{\mathrm{s}}-V_{\mathrm{min}}$ détermine le nombre d'électrons susceptibles d'atteindre la grille d'extraction. Le franchissement de cette barrière est non seulement conditionné par l'énergie initiale mais encore par la répartition angulaire des électrons émis. $\mathrm{La}$ figure 4 montre des trajectoires d'électrons pour différentes énergies initiales d'émission et pour différents angles de sortie. Lorsque l'énergie initiale est supérieure à $U_{\text {so }}$ tous les électrons franchissent la barrière de potentiel.

La figure 5 représente le même phénomène pour un champ d'extraction différent. Ici la barrière de potentiel a été abaissée et les électrons de plus faible énergie peuvent la franchir. La figure 6 met bien en évidence l'effet d'abaissement de barrière sur la collection. Ces diagrammes d'acceptance caractérisent la collection et permettent de connaître l'ensemble des énergies et angles d'émission des électrons secondaires qui parviennent à atteindre la grille. La limite inférieure en énergie correspond à cette barrière de potentiel. 

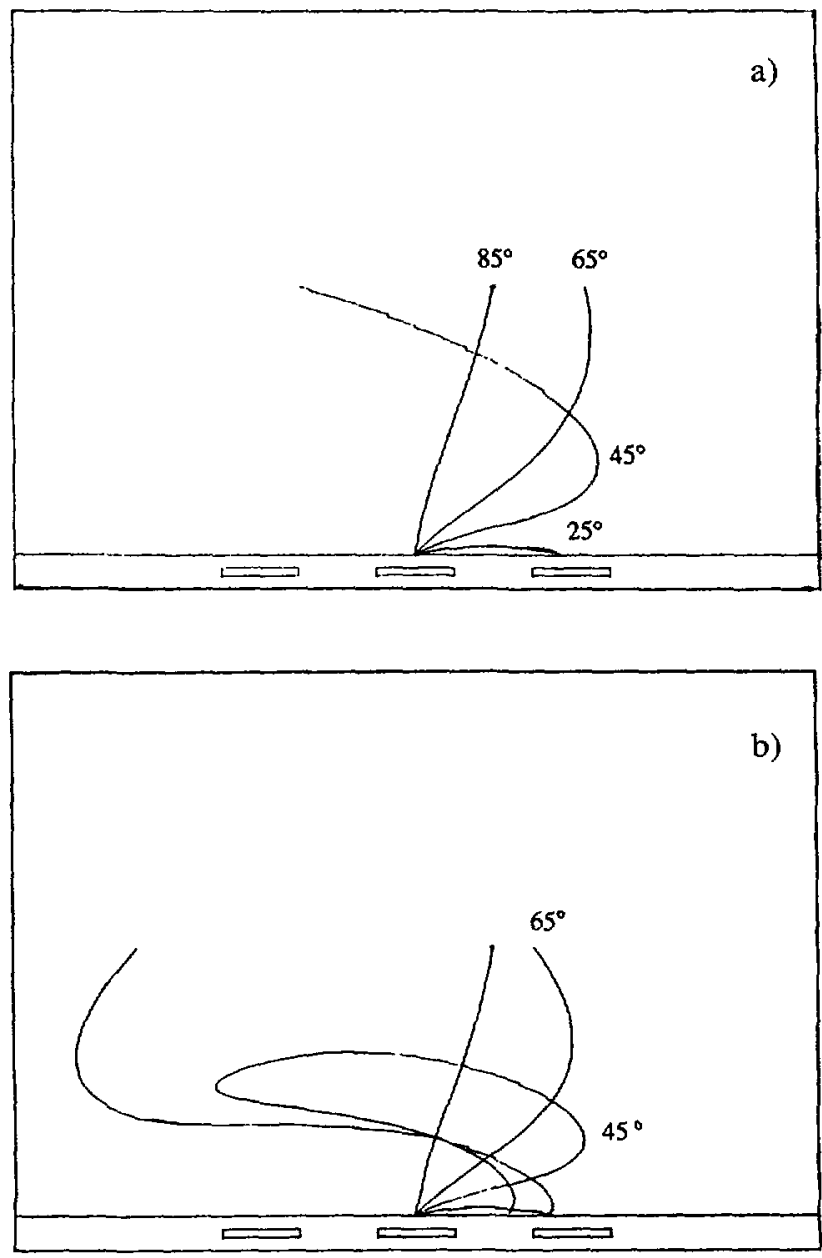

Fig. 4. - Trajectoires des électrons secondaires pour un champ d'extraction de $10 \mathrm{~V} / \mathrm{mm}$. Les variables sont l'angle de sortie des électrons $25^{\circ}, 45^{\circ}, 65^{\circ}, 85^{\circ}$ et leurs énergies a) $\left.3,9 \mathrm{eV} \mathrm{b}\right) 4,0 \mathrm{eV}$. La barrière de potentiel est de $3,8 \mathrm{~V} . V_{\mathrm{s}}=4,47 \mathrm{~V} V_{\mathrm{min}}=0,69 \mathrm{~V}$.

[Secondary electron trajectories for an Extraction field of $10 \mathrm{~V} / \mathrm{mm}$. The variables are electron emission angle $25^{\circ}, 45^{\circ}, 65^{\circ}, 85^{\circ}$ and their energies a) $3.9 \mathrm{eV}$ b) $4.0 \mathrm{eV}$. The potential barrier is $3.8 \mathrm{~V}$. $\left.V_{\mathrm{s}}=4.47 \mathrm{~V} V_{\min }=0.69 \mathrm{~V}.\right]$

Le courant détecté par l'analyseur est modulé par le champ d'extraction et le nombre d'électrons émis retournant à la surface. La courbe de la figure 7 , pour une configuration dans laquelle la piste centrale est enterrée, montre que le potentiel de surface croît très peu avec le champ d'extraction. Le potentiel de surface ne peut favoriser seul l'accumulation de charges en fonction de l'augmentation de la tension d'extraction. Le bilan des courants donne :

$$
I_{0}=I_{\mathrm{s}}+I_{\mathrm{b}}+I_{\mathrm{c}} \text { avec } I_{\mathrm{s}}=\delta I_{0}
$$

où $\delta$ est le coefficient d'émission secondaire $(\sigma=\delta+\eta)$ avec $\sigma$ rendement total d'émission des électrons, $I_{0}$ le courant du faisceau primaire, $I_{\mathrm{s}}$ le courant d'électrons secondaires émis du matériau, $I_{\mathrm{b}}$ le courant des électrons secondaires et $I_{\mathrm{c}}$ le courant de charge du circuit. 

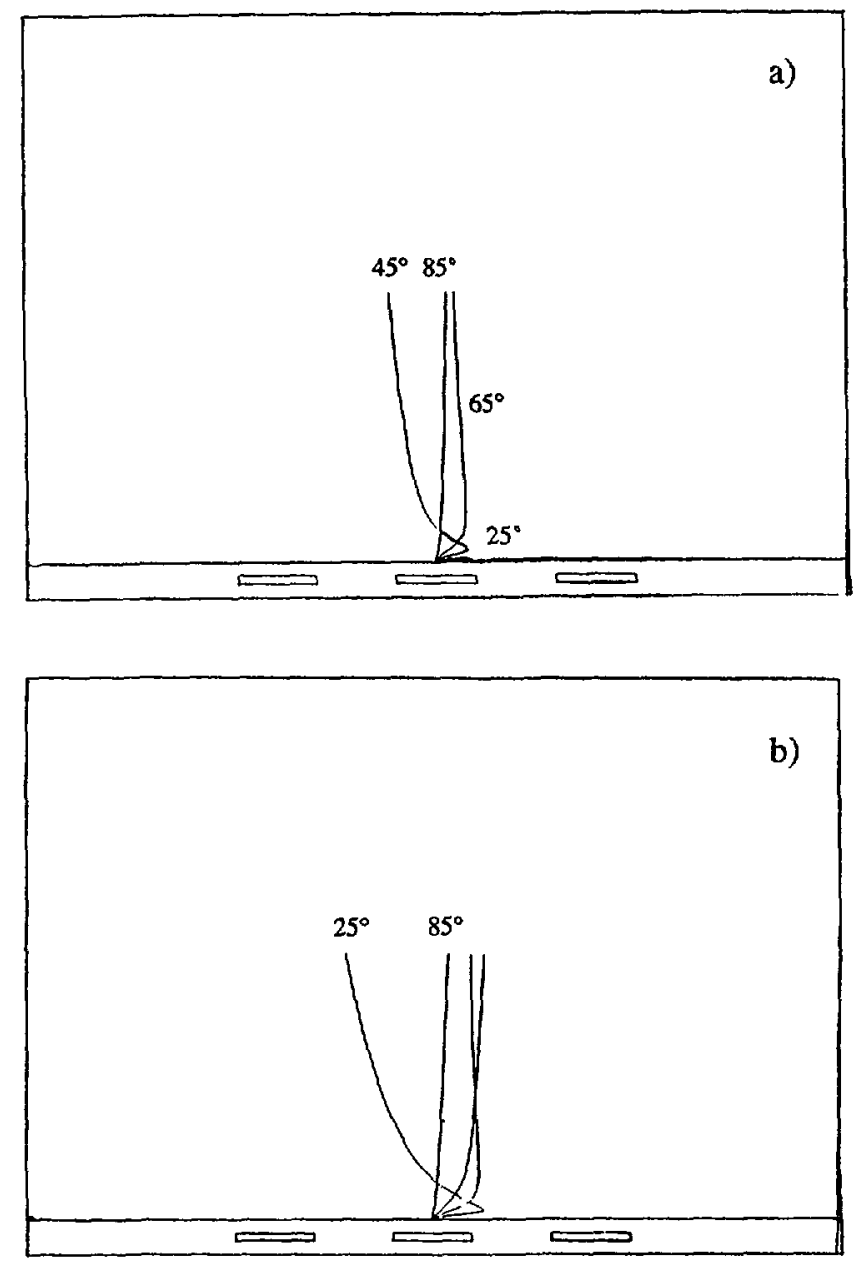

Fig. 5. - Trajectoires des électrons secondaires pour un champ d'extraction de $1000 \mathrm{~V} / \mathrm{mm}$. Les variables sont l'angle de sortie des électrons $25^{\circ}, 45^{\circ}, 65^{\circ}, 85^{\circ}$ et leurs énergies a) $\left.0,2 \mathrm{eV} \mathrm{b}\right) 0,6 \mathrm{eV}$. La barrière de potentiel est de $0,08 \mathrm{~V} . V_{s}=4,73 \mathrm{~V} V_{\min }=4,65 \mathrm{~V}$.

[Secondary electron trajectories for an Extraction field of $1000 \mathrm{~V} / \mathrm{mm}$. The variables are the electron emission angles $25^{\circ}, 45^{\circ}, 65^{\circ}, 85^{\circ}$ and its energies a) $\left.0.2 \mathrm{eV} \mathrm{b}\right) 0.6 \mathrm{eV}$. The potential barrier is $0.08 \mathrm{~V}$. $\left.V_{\mathrm{s}}=4.73 \mathrm{~V} V_{\operatorname{man}}=4.65 \mathrm{~V}.\right]$

Ce courant de charge peut être exprimé par :

$$
I_{\mathrm{c}}=I_{\mathrm{ch}}+I_{\mathrm{r}}\left(V_{\mathrm{ext}}\right)
$$

où $I_{\text {ch }}$ est le courant de conduction qui pénètre directement le matériau et $I_{\mathrm{r}}$ est le courant redistribué et dépend directement du potentiel d'extraction. L'énergie primaire du faisceau d'électrons choisie est prise légèrement inférieure à l'énergie du second cross-over. Cette énergie favorise la charge positive en surface du circuit en donnant un rendement entre les électrons primaires et secondaires supérieur à 1 . Grâce au courant de redistribution cette charge restera petite pour une faible tension d'extraction alors qu'elle pourra être importante pour une tension élevée. 


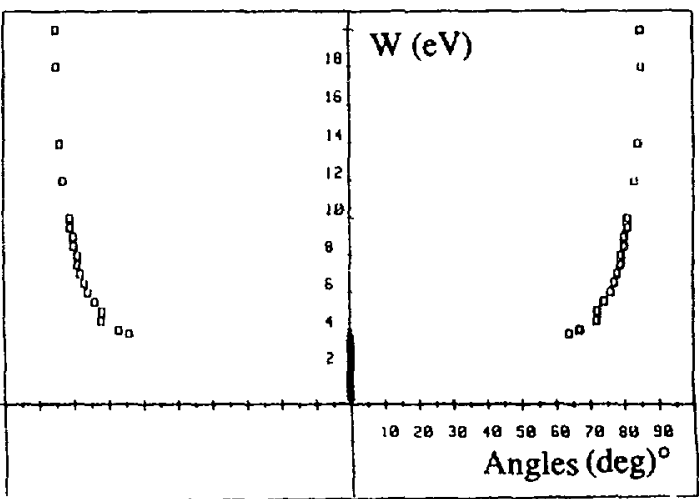

a)

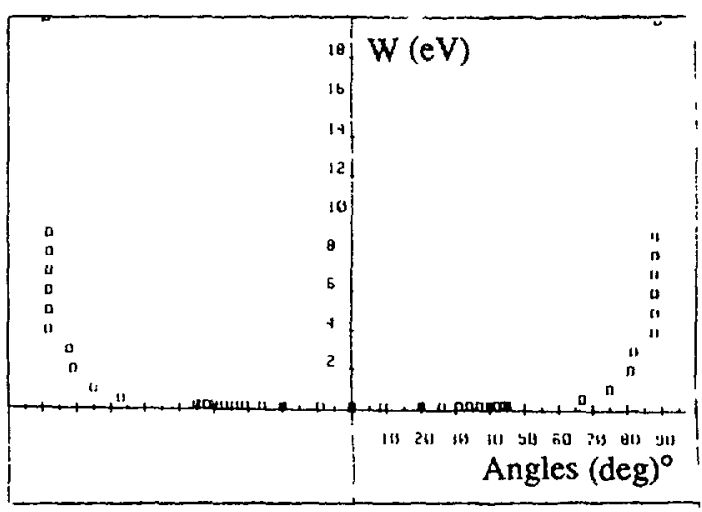

b)

Fig. 6. - Diagramme d'acceptance pour une configuration (0-5-0) $\mathrm{V}$ et un champ d'extraction de $10 \mathrm{~V} / \mathrm{mm}$ a) et $1000 \mathrm{~V} / \mathrm{mm} \mathrm{b}$ ). L'angle de collection des electrons secondaires sur la figure est défini comme l'ensemble des angles d'émission des électrons mesurés à partir de l'horizontale et qui sont collectés par le détecteur. La limite inférieure en énergie correspond à la barrière de potentiel.

[Acceptance diagram for a configuration (0-5-0) V and an Extraction field for a) $10 \mathrm{~V} / \mathrm{mm}$ and for b) $1000 \mathrm{~V} / \mathrm{mm}$. The SE collection angle in this figure is defined as the range of emission angles measured from the horizontal and which are collected by the detector. The lower energy limit correspond to the potential barrier.]

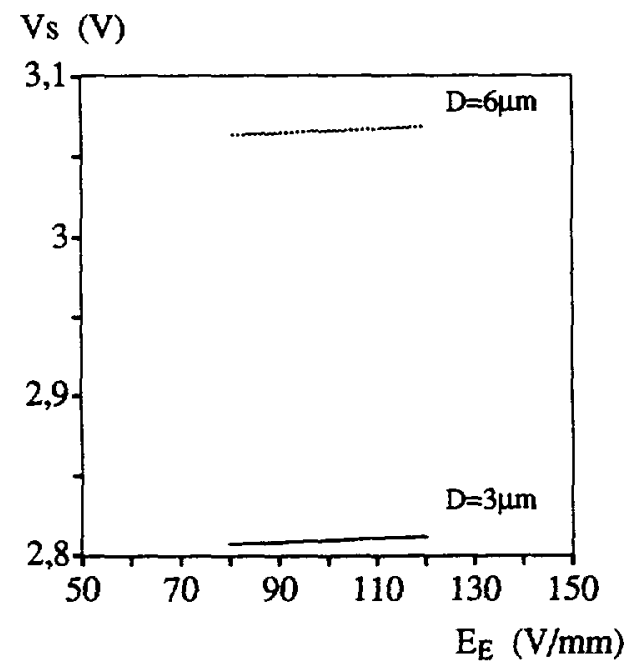

Fig. 7. - Variation du potentiel de surface en fonction du champ d'extraction appliqué pour $W=2,4 \mu \mathrm{m}, D=3 \mu \mathrm{m}$ et $D=6 \mu \mathrm{m}$.

[Variation of the surface potential as a function of the extraction field for $W=2.4 \mu \mathrm{m}, D=3 \mu \mathrm{m}$ et $D=6 \mu \mathrm{m}$.

\section{Autres conséquences.}

Le logiciel permet de plus d'étudier les temps de transit [12], c'est-à-dire de vérifier que lors d'un changement de potentiel rapide de la piste, les électrons secondaires émis de l'échantillon subissent les effets dus à la variation du champ électrique dans l'espace. La résolution 


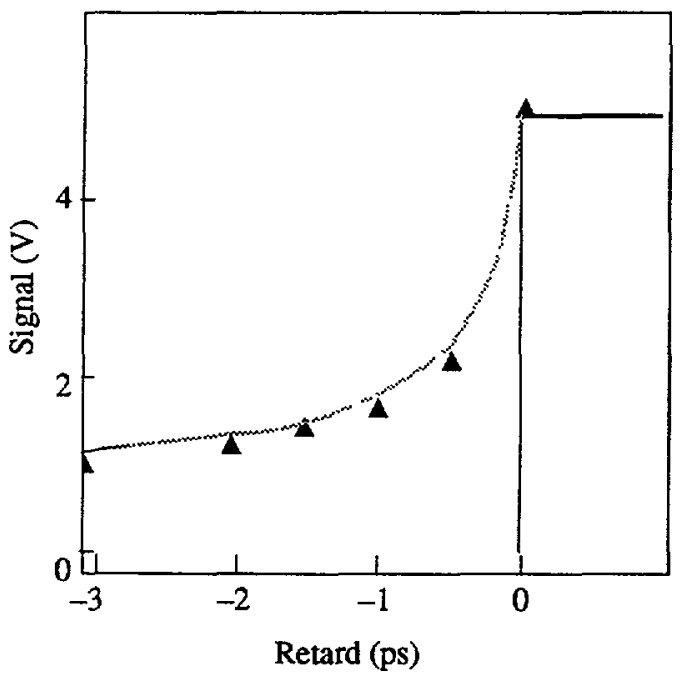

Fig. 8. - Distortion du signal en fonction du temps.

[Distorted waveform as a function of time.]

temporelle des signaux à mesurer est ainsi limitée par cet effet « temps de transit », c'est-à-dire limitée par le temps requis par les électrons secondaires pour traverser la région au-dessus du point de test. La variation rapide du potentiel des pistes modifie dans ce cas la trajectoire des électrons émis et leur énergie de collection. La figure 8 montre la distorsion du temps de montée du signal lors d'un changement rapide du potentiel.

\section{Conclusion.}

La méthode numérique de résolution de l'équation de Poisson appliquée aux cas des interconnexions de circuits intégrés passivés en configuration de test par faisceau d'électrons permet de déterminer l'effet du champ d'extraction sur les trajectoires des électrons et d'en déduire le courant détecté. De plus la connaissance de ces trajectoires facilite l'interprétation du phénomène de collection des électrons secondaires. Elle permet aussi l'optimisation des paramètres du MEB qui conditionnent la mesure du potentiel de surface.

\section{Bibliographie}

[1] Cole E. I., Bagnell Jr. C. R., Scanning Microscopy 2 (1988) 133-150.

[2] Mizuno F., Mori S., J. Vac. Sci. Technol. 19 (4) (1981).

[3] Wolfgang E., Linder R., Fazekas P., IEEE Trans. Elec. Dev. 26 (1979) 549-559.

[4] REINERS W., Scanning Electron. Microsc. 2 (1988) 161-175.

[5] Gorlich S., Hermann K. D., Kubalek E., Microcircuit Eng. (1984) 451-460.

[6] Miyoshi M., Ishikawa M. and Okumura K., Proceedings Annual Conference on Scanning Electron Microscopy (SEM inc., O'Hare, IL) (1982) pp. 1507-1514.

[7] GiRard P., Scanning Microscopy 2 (1988) 151.

[8] Roche F. M., Bocus S. D., Proceeding of the $3^{\text {rd }}$ EOBT'91, COMO, Microelectronic Eng. 16 (1992) 157-164.

[9] Menzel E., KubaleK E., Scanning 5 (1983) 103-122.

[10] Nougier J.-P., Méthodes de Calcul Numérique (Masson, France) pp. 216-272.

[11] Chim W. K., Low T. S., Chan D. S. H. and Phang J. C. H., J. Phys. D. . Appl. Phys. 21 (1988) 1 9.

[12] Nakamae K., Fujoka H. and Ura K., Meas. Sci. Technol. 1 (1990) 894-902. 